\title{
VALORES REFERENCIAIS DA ANÁLISE DO LÍQUIDO PERITONEAL DE EQÜINOS SADIOS
}

\author{
REFERENCE VALUES FOR ANALYSIS OF PERITONEAL \\ FLUID OF HEALTHY EQUINES
}

\author{
Mariana Machado Neves ${ }^{1}$ Antônio de Pinho Marques Jr. ${ }^{2}$ \\ Geraldo Eleno Silveira Alves ${ }^{2}$ Evenilde Picardi Faria ${ }^{2}$
}

\section{RESUMO}

\begin{abstract}
Considerando a casuística de distúrbios abdominais em eqüinos, e o fato da paracentese ser uma técnica fácil e segura para o animal, a análise do líquido peritoneal torna-se um importante exame auxiliar no diagnóstico e direcionamento da conduta clínica. Como no Brasil não existem valores de referência desse exame em eqüinos sem raça definida, este trabalho objetivou o estabelecimento desses valores para esses animais. Foram usados sessenta eqüinos sem raça definida, procedentes da Região Metropolitana de Belo Horizonte-MG, os quais foram vermifugados e mantidos na Escola de Veterinária da UFMG, recebendo a mesma alimentação. Foi realizada paracentese abdominal, para obtenção de amostra fracionada em três alíquotas: uma contendo EDTA, outra citrato de sódio e outra sem anticoagulante. A alíquota sem anticoagulante foi subdividida para se fazer esfregaços em lâminas para contagem diferencial de leucócitos e para dosagem da proteína total. Na alíquota com citrato, foi dosado fibrinogênio e na com EDTA foi feita contagem total de leucócitos. Os resultados obtidos foram: Leucócitos

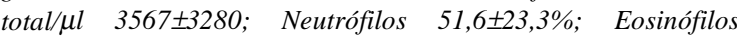

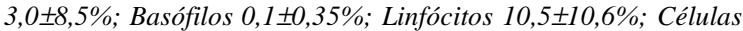

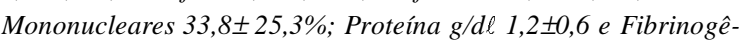
nio $\mathrm{g} / \mathrm{d} \ell 0,05 \pm 0,015$.
\end{abstract}

Palavras-chave: paracentese, líquido peritoneal, eqüino e bioquímica.

\section{SUMMARY}

The high casuistic of abdominal disturbs in equine, and the knowledge that paracentesis is an efficient technique, simple and safe for the animal, result that the analysis of the peritoneal fluid becomes an important aid for diagnosis and clinical orientation in individual cases. As in Brazil there are no reference values for peritoneal fluid evaluation for working horses, this work intends to provide some values for that category of animals. Sixty healthy working horses, from the Metropolitan area of Belo Horizonte City, Minas Gerais State, Brazil were used. The animals were maintained in the Veterinary Hospital of the Veterinary School of the Federal University of Minas Gerais Brazil, kept under the same nutricional and environmental conditions. Abdominal paracentesis was performed to obtain a sample in three aliquots: with EDTA, with sodium citrate and without anticoagulant substance. The aliquot without anticoagulant was subdivided to prepare smears in glasses to make leukocyte differentiation, cytological examination and total protein determination. In the aliquot with sodium citrate was determined fibrinogen and in the one with EDTA was made total leukocytes count. The results were Total Leukocytes/ $\mathrm{ll}$

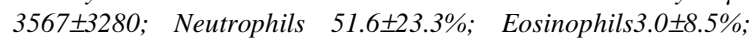
Basophils 0.1 $\pm 0.35 \%$; Lymphocytes $10.5 \pm 10.6 \%$; Mononucleated

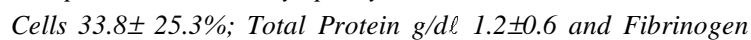
g/d $\ell 0.05 \pm 0.015$.

Key words: paracentesis, peritoneal fluid, equine, biochemistry.

\section{INTRODUÇÃO}

Os testes laboratoriais são um apoio importante no diagnóstico de problemas clínicos na prática veterinária. Os exames nos laboratórios de Patologia Clínica devem ser acessíveis, confiáveis, rápidos e de baixo custo, fornecendo para o médico veterinário tabelas próprias de valores referenciais, uma vez que existem variáveis que afetam esses valores, incluindo a espécie, a idade e o sexo do

\footnotetext{
${ }^{1}$ Bolsista de Iniciação Científica, Departamento de Clínica e Cirurgia Veterinárias, Escola de Veterinária, Universidade Federal de Minas Gerais (UFMG), Av. Antônio Carlos, 6627, 31270-010, Belo Horizonte, MG. E-mail: carpedie@ net.em.com.br. Autor para correspondência.

${ }^{2}$ Departamento de Clínica e Cirurgia Veterinárias, Escola de Veterinária, UFMG 
animal. Dispondo-se dessas tabelas, minimizam-se influências devido a equipamentos e à metodologia própria de cada rotina (MESSER, 1995).

A obtenção do líquido peritoneal por meio da paracentese abdominal, considerada uma prática fácil, segura para o animal e de baixo custo, é o teste laboratorial mais esclarecedor para auxiliar a classificação do tipo de doença e também para determinar a severidade da lesão abdominal (TULLENERS, 1983), tornando a análise de líquido peritoneal um exame importante e útil não só para o diagnóstico, mas também para o prognóstico e para o direcionamento da conduta clínica em eqüinos (MESSER, 1995).

Valores referenciais para o líquido peritoneal têm sido descritos para algumas raças de eqüinos em diversos países. No Brasil, existem poucas referências específicas (MENDES,1995; MACORIS,1995) para raças e não existe ainda referência para o exame do líquido peritoneal em eqüino sem raça definida, sendo pois, o objetivo deste trabalho estudar e estabelecer esses valores de referência.

\section{MATERIAL E MÉTODOS}

Foram usados sessenta eqüinos sem raça definida, com variação de idade, sexo e procedência, que foram vermifugados e mantidos no Hospital Veterinário da Escola de Veterinária da UFMG, sob regime alimentar padronizado. Foi realizada a paracentese abdominal, segundo a técnica de WHITE (1990) para obtenção de uma amostra fracionada em três alíquotas: uma contendo EDTA, outra contendo citrato de sódio e outra sem anticoagulante.

O líquido peritoneal foi avaliado macroscopicamente, observando-se o aspecto, a coloração e a presença ou ausência de coágulos. As avaliações celulares foram realizadas na amostra com EDTA e, numa alíquota da amostra sem anticoagulante. $\mathrm{Na}$ amostra com EDTA, foi feita contagem do número total de leucócitos pelo contador automático da marca Celm, enquanto na amostra sem anticoagulante foram feitos esfregaços em lâminas, para contagem diferencial de leucócitos corados pela técnica de May-Grunwald Giemsa, além de outros aspectos. A população de células foi classificada em neutrófilos, eosinófilos, basófilos, linfócitos e células mononucleares (monócitos e macrófagos). A amostra contendo citrato de sódio foi utilizada para dosagem de fibrinogênio pelo método de Menzie, enquanto que a amostra sem anticoagulante foi utilizada para dosagem de proteínas totais pelo método do Biureto.

\section{RESULTADOS}

O líquido peritoneal, na análise macroscópica das amostras, apresentou cor amarelo-palha e aspecto límpido a ligeiramente turvo, caracterizando um líquido normal. Os resultados encontrados estão demonstrados na tabela 1 .

\section{DISCUSSÃO}

A técnica de paracentese abdominal mostrou-se prática, segura e eficiente, à semelhança do também relatado por TULLENERS (1983) e WHITE (1990). A média da contagem dos leucócitos totais apresentou-se dentro dos limites estabelecidos para outras raças de eqüinos (BACH, 1973; COFFMAN, 1973; BACH \& RICKETTS, 1974). A contagem diferencial de leucócitos se mostrou similar à de outros trabalhos, caracterizado por número maior de neutrófilos (BACH \& RICKETTS, 1974; MCGRATH, 1975; BROWNLOW et al., 1981) e menor número de linfócitos (BACH \& RICKETTS, 1974; GORDON \& WILSON, 1987). O valor para eosinófilos foi superior às médias descritas por COFFMAN (1980); BACH \& RICKETTS (1974), sendo que a maior média e desvio padrão provavelmente tenham ocorrido devido à presença de eosinofilia no líquido peritoneal de dois eqüinos, possivelmente em decorrência de infestação parasitária, apesar desses animais terem sido vermifugados. A média verificada para os basófilos foi semelhante às descritas por BROWNLOW et al. (1981) e PARRY \& BROWNLOW (1992). Os valores encontrados para células mononucleares estão nos limites considerados normais, segundo BACH (1973) e BACH \& RICKETTS, (1974).

Os valores de proteína total, em torno de $1,2 \mathrm{~g} / \mathrm{d} \ell$, assemelham-se aos descritos na literatura (BACH \& RICKETTS, 1974; BROWNLOW et al., 1981). Na dosagem do fibrinogênio, a técnica de

Tabela 1 - Valores de proteína total, fibrinogênio e contagem global e diferencial de leucócitos no líquido peritoneal de eqüinos sem raça definida.

\begin{tabular}{|c|c|c|}
\hline Concentração de Proteínas Totais $(\mathrm{g} / \mathrm{d} \ell)$ & $1,2 \pm 0,6$ & \\
\hline Concentração de Fibrinogênio (g/d $\ell$ ) & $0,05 \pm 0,015$ & \\
\hline Número Total de Leucócitos $\left(/ \mathrm{mm}^{3}\right)$ & $3567 \pm 3280$ & \\
\hline Contagem Diferencial de Leucócitos: & Valor relativo & Valor absoluto \\
\hline Neutrófilos & $51,6 \pm 23,3$ & $1841 \pm 831$ \\
\hline Linfócitos & $10,5 \pm 10,6$ & $375 \pm 21,4$ \\
\hline Basófilos & $0,1 \pm 0,35$ & $4 \pm 12$ \\
\hline Eosinófilos & $3,0 \pm 8,5$ & $107 \pm 303$ \\
\hline Células Mononucleares & $33,8 \pm 25,3$ & $1206 \pm 902$ \\
\hline
\end{tabular}


precipitação com solução saturada de sulfato de amônio demonstrou eficiência e confiabilidade, permitindo mensurações inferiores a $0,1 \mathrm{~g} / \mathrm{d} \ell$, que é o limite inferior da técnica de precipitação por aquecimento, utilizada pela maioria dos autores. A concentração de fibrinogênio nas amostras estudadas mostrou-se dentro dos limites fisiológicos descritos por GORDON \& WILSON (1987).

\section{REFERÊNCIAS BIBLIOGRÁFICAS}

BACH, L.G. Exfoliative citology of peritoneal fluid in the horse Veterinary Annual v.1, p.102-109, 1973.

BACH, L.G., RICKETTS, S.W. Paracentesis as an aid to the diagnosis of abdominal disease in the horse. Equine Veterinay Journal v.6, n.3, p.116-121, 1974.

BROWNLOW., M.A, HUTCHINS, D.R, JOHNSTON, K.G Reference values for equine peritoneal fluid. Equine Veterinary Journal, v.13, n.2, p.127-130, 1981.

COFFMAN, J.R. Technic and interpretation of abdominal paracentesis. Modern Veterinary Practice, v.54, p.79-81, 1973.

COFFMAN, J.R. Peritoneal fluid. Veterinary Medicine Small Animal Clinical, v.75, n.8, p.1285-1288, 1980.
GORDON, B., WILSON, J. Equine colic: interpreting the diagnostic tests. Veterinary Medicine, v.82, n.6, p.640-645, 1987.

MACORIS, D.G. Importância da avaliação do líquido peritoneal no diagnóstico e prognóstico da cólica. In: CICLO INTERNACIONAL DE CÓLICA EQÜINA, 2, 1995, Jaboticabal. Anais... Faculdade de Ciências Agrárias e Veterinárias - UNESP, 1995. 54 p. p.21-23.

MENDES, L.C.N. Estudo das alterações clínicas e laboratoriais de eqüinos submetidos à peritonite experimental. In: CICLO INTERNACIONAL DE CÓLICA EQÜINA, 2, 1995, Jaboticabal. Anais... Jaboticabal : Faculdade de Ciências Agrárias e Veterinárias, UNESP, 1995. 54p. p.48 -50.

MESSER, N.T. The use of laboratory tests in equine practice. Veterinary Clinical North American: Equine Pratice, v.11, n.3, p.345-350, 1995.

PARRY, B.W., BROWNLOW., M.A. Peritoneal fluid. In: COWELL, R.L., TYLER, R.D. Cytology and hematology of the horse. Santa Bárbara : American Veterinary Publications, 1992. p.121-151.

TULLENERS, E.P. Complications of abdominocentesis in the horse. Journal American Veterinary Medicine Association, Schaumburg, v.182, n.3, p.232-234, 1983.

WHITE, N.A. Determining the diagnosis and prognosis of the acute abdominal. In : WHITE, N.A. The equine acute abdomen. Philadelphia : Lea \& Fabiger, 1990. p. 101-140.

Ciência Rural, v.30, n.5, 2000. 